\title{
Endometriosis and gynaecological cancers: molecular insights behind a complex machinery
}

\author{
Vaclav Vetvicka ${ }^{1}$, Ludek Fiala², Simone Garzon ${ }^{3}$, Giovanni Buzzaccarini ${ }^{4}$, Milan Terzic $^{5,6,7}$, \\ Antonio Simone Laganà ${ }^{8}$
}

${ }^{1}$ Department of Pathology, University of Louisville, Louisville, KY, United States

${ }^{2}$ Institute of Sexology, First Faculty of Medicine, Charles University, Prague, Czech Republic

${ }^{3}$ Department of Obstetrics and Gynecology, AOUI Verona, University of Verona, Verona, Italy

${ }^{4}$ Department of Women and Children's Health, University of Padua, Padua, Italy

${ }^{5}$ Department of Medicine, School of Medicine, Nazarbayev University, Nur-Sultan, Kazakhstan

${ }^{6}$ Clinical Academic Department of Women's Health, National Research Center of Mother and Child Health, University Medical Center,

Nur-Sultan, Kazakhstan

${ }^{7}$ Department of Obstetrics, Gynecology and Reproductive Sciences, University of Pittsburgh School of Medicine, Pittsburgh, PA, United States

${ }^{8}$ Department of Obstetrics and Gynecology, "Filippo Del Ponte" Hospital, University of Insubria, Varese, Italy

\begin{abstract}
Endometriosis is described as the presence of both endometrial glandular and stromal cells outside the uterine cavity. A major characterization of this disease is ectopic implantation of endometrial cells with increased migration. It is one of the leading causes of morbidity among premenopausal women, with a prevalence of $10-16 \%$ of women of reproductive age. Despite over century of intensive research, none of the current treatment options represents a real cure. Based on the current knowledge, endometriosis, particularly its atypical version, is considered to be a transitional form from benign disease to tumour. However, the exact mechanisms of this conversion are still not fully established.
\end{abstract}

Key words: endometriosis, cancer, ovarian cancer.

\section{Introduction}

Endometriosis is a complex and multifactorial disease, in which diagnosis, markers, and therapeutic treatments remain unclear and somehow enigmatic [1]. The fact that no single theory about the pathology and causes of endometriosis is currently accepted [2, 3] explains the lack of progress. For more details about pathogenesis of endometriosis, see [4].

Currently, there is no doubt that endometriosis is a benign disease; however, more and more studies support the notion that it may represent a condition that could lead to the development of pre-cancerous lesions. The first report of a suspected malignant change in endometriosis occurred almost 100 years ago [5] with the observation of malignant changes in the endometrial tissue. This hypothesis was confirmed more than 25 years later [6]. Atypical endometriosis is often considered a transitional form from benign disease to cancer [7]. However, the current classification of endometriosis into typical and atypical is more important for diagnosis than for clinical practice. The problem is further elevated by the findings that the possible association between cancer and endometriosis varies according to the histologic subtype of ovarian cancer [8].
This hypothesis is not only based on the known association between endometriosis and cancer, but also on numerous common features shared by endometriosis and cancer, such as the ability to evade apoptosis, stem cell-like dysregulation, neovascularization, cancer-reminting implantation at distant sites, and strong effects on the immune system [4]. However, the question of whether atypical endometriosis really is a precursor of future ovarian cancer has never been satisfactorily answered. Lately, a discussion suggesting that endometriosis is not a benign disease but an invasive one [9] occurred, but despite several points supporting this option, it is still not accepted by the scientific community. Possible relationships between endometriosis

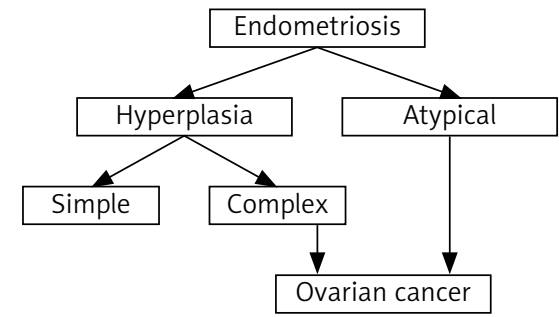

Fig. 1. Possible relation between endometriosis and development of ovarian cancer 


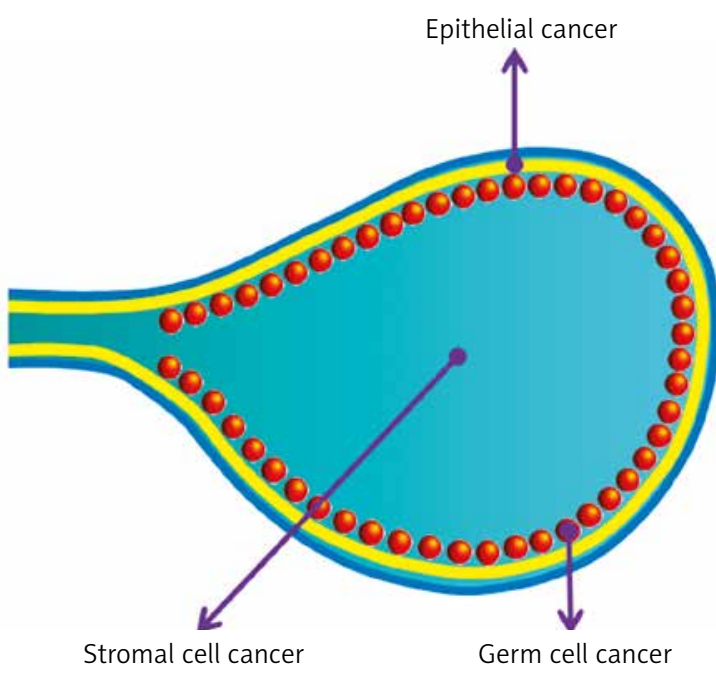

Fig. 2. Basic types of ovarian cancer

and the development of ovarian cancer are shown in Figure 1.

Women with endometriosis appear to be more likely to develop certain types of cancer, particularly ovarian, non-Hodgkin's lymphoma, and brain tumours [10-12]. The association between endometriosis and breast cancer is still contradictory [11]. Similarly, a review of the literature suggested a link between endometriosis and ovarian cancer, but not breast and cervical cancer [13], so these possible links are even more questionable.

Some cases, despite usually being case reports, underline the complexity of the endometriosis-cancer relationship. A case of primary endometrioid carcinoma arising from deep infiltrating endometriosis 6 years after diagnosis of ovarian cancer [14] can serve as an example. Similar case reports do not provide any meaningful information about the mechanisms of action or pathology of cancer development, but strongly suggest the need for long-term follow-up for survivors of ovarian cancer with co-existent endometriosis.

Epigenetic changes such as the presence of some mRNAs or DNA methylation have been suggested. One of the possible triggers of conversion from benign to malignant disease might be microRNAs (miRNAs) [15]. One of the suggestions was offered by Nakamura et al. [16]. However, even though a high level of miR-486-5p in serum and its upregulation causing migration and proliferation of ovarian endometrioma cells seems to support their hypothesis, the real proof was missing. Another study found 23 individual miRNAs expressed differently in healthy women and in women with endometriosis and/or ovarian cancer [17].

Comprehensive miRNA profiling from ovarian cancer and its associated endometriosis showed that the expressions of miRNAs were significantly different [18]. Based on similar studies, miRNAs have been suggested as a marker of either disease or endometriosis-cancer progression. With limited information and a high number of individual miRNAs, it is not currently possible to ascertain whether miRNAs are really relevant and if so, which ones.

Mutation of ARID1A, which is a tumour suppressor gene involved in endometriosis-cancer transformation [10]. The fact that tumours with a mutated ARID1A gene often have a better higher survival rate might explain why endometrium-associated ovarian cancer often has lower mortality than other types of ovarian cancer. The relatively wide range of this mutation in ovarian endometroid carcinoma (30-48\%) might explain the often contradictory results of numerous studies. Additional studies have suggested the role of CTNNB1 and PTEN genes, PIK3-AKT-mTOR pathway, methylation of the ER promoter, high levels of oestrogen, and inflammation $[19,20]$.

\section{Ovarian cancer}

The association between endometriosis and cancer is focused mostly on ovarian cancer, most of all endometroid and clear-cell ovarian cancer subtypes [21-25]. Basic types of ovarian cancer are shown in Figure 2.

Most studies agree that endometriosis and ovarian cancer are somehow linked, but the relationship is not clear and is often controversial. A study of operated ovarian cancer patients showed $10 \%$ with coexisting ovarian endometriosis. This number increased to $36.8 \%$ in patients with clear cell ovarian cancer

Histopathological studies have suggested that atypical endometriosis is a transition between endometriosis and cancer [26]. If true, endometriosis is a premalignant condition. This hypothesis is supported by the fact that almost $70 \%$ of endometriosis-associated ovarian carcinomas occur in the presence of atypical ovarian endometriosis. Clinically significant similarities between endometriosis and ovarian cancer exist, some of which can influence the incidence rate of cancer. Infertility or late menopause can serve as examples of clinical manifestations related to increased risk [27]. On the other hand, factors such as hysterectomy, use of oral contraceptives, or tubal ligation surprisingly result in lower risk of cancer development [28].

One of the new areas is focused on the possibility that endometrial cysts might be the origin of endometriosis-associated ovarian cancer (EAOC), but this hypothesis is only discussed rather than confirmed. The current hypothesis assumes that the process involves DNA damage and instability in endometrial cysts followed by selection of cells with high antioxidant capacity [29]. The evidence of resistance of ovarian cancer to oxidative stress is lacking. Some studies have suggested that this type of cancer is probably caused by eutopic endometrial glandular cells with oncogenic mutation upon engrafting in the ovary and not by endometrial cysts undergoing oncogenic mutations [30]. Most of the research believes that endometrial cysts are not the cause of ovarian can- 
cer. Possible steps involved in changes from endometriosis to EAOC are summarized in Figure 3.

On the one hand, a large case-control study demonstrated that the lifetime chance of ovarian cancer was $50 \%$ higher among women suffering from endometriosis, but the possible triggering mechanisms such as oral contraception use or the number of births showed no effects. A subsequent review study revealed up to 3.5-fold higher development of endometrioid and clear cell tumours among these women [31]. On the other hand, a systematic review of 25 years of studies showed only modest effects, ranging between 1.3 and 1.9 [32]. The largest study completed by Olson [33] with an average 13-year follow-up did not find any difference in ovarian cancer occurrence between normal patients and patients with endometriosis. A recent review comparing individual studies showing either high or no incidence of endometriosis-ovarian cancer relation was done by Pejovic et al. [34].

Despite often cited relations between endometriosis and ovarian cancer, the overall frequency was found to be 0.3 to $0.8 \%$ [35]. Conversely, numerous other studies found much higher risk - from 19\% [36] to a 4-fold increase [37]. More detailed studies tried to elucidate the reasons behind these contradictory results. An evaluation of 200,000 patients showed that higher risk is associated with greater age, pelvic inflammatory problems, and being childless [38]. A retrospective study of malignancy risks in endometriosis found than not only ovarian cancer, but also cervical, breast, and thyroid cancer have higher incidence [39]. If confirmed, cancer screening might be beneficial to all women over 40 years of age originally diagnosed with endometriosis. However, other studies found no increased coincidence of breast cancer [20]. Strangely, the association, often significant, has been found in older studies, whereas the newest population-based cohort studies found no association [19, 40].

Another controversy exists with prognosis. Ovarian cancer has the highest mortality of all gynaecological cancers, but several studies found that patients with both endometriosis and ovarian cancer have better prognosis [41]. Even more studies, however, did not confirm these findings. The problems might be caused by a low number of patients in the studies and over-reliance on self-reporting. To answer this question, a large cohort study evaluating over 32,000 women with a diagnosis of ovarian cancer over a 25-year interval was performed. The results confirmed that patients with histologically confirmed endometriosis and ovarian cancer have a longer overall survival rate [21, 22]. Because the aim of this study was to find only possible differences in prognosis, we still have no clue about the possible mechanisms. Clearly, more studies are necessary. Possible stratification by endometriosis status might help to elucidate the role of endometriosis.

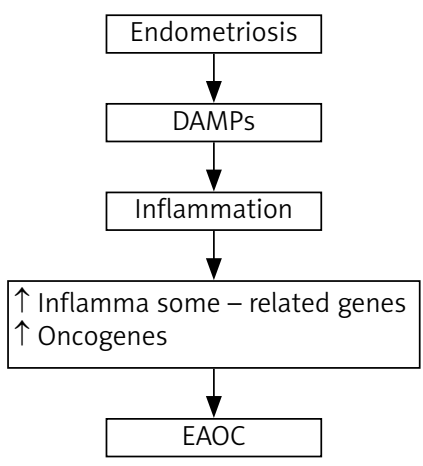

Fig. 3. Possible steps involved in changes from endometriosis to endometriosis-associated ovarian cancer

Another unclear association is the survival prognosis. Indeed, ovarian cancer resulting from endometriosis has some special characteristics such as having endometroid or clear cell histology and a better prognosis [42]. However, the question of endometriosis being a prognostic factor for cancer survival is not clear. On the one hand, some studies found no definitive association between the presence of endometriosis and survival [43]. On the other hand, another study found significantly better survival in women with endometriosis than for all malignancies combined [44]. From the genetic point of view, the malignant transformation of endometriosis to ovarian cancer might be triggered by some essential genes. A recent study used RNA sequencing of several types of tissues ranging from normal endometriosis to atypical endometriosis and ovarian cancer and found significant significantly increased levels of mRNA of tetraspanin 1 [45]. These data were confirmed by evaluation of tetraspanin 1 protein levels. The authors suggest that overexpression of this gene enhanced cell proliferation and invasion, probably via increasing activity of AMP-activated protein kinase. If confirmed, tetraspanin 1 might be used first as a marker in screening for the risk of endometriosis-ovarian cancer conversion and later as a therapeutic target. The additional 13 genes regulated during the transition phases might also be important, but their possible role is still unclear. The most important genes involved in development of endometriosis, EAOC, and ovarian cancer are shown in Figure 4.

Another possibility might be the recently reported molecular pathways associated with ARID1A mutations, which might be involved in the progression from endometriosis to atypical endometriosis and subsequent EAOC [46]. This hypothesis is based on the finding of ARID1A mutation occurring in app. $50 \%$ of endometriosis-associated ovarian cancer [47]. A meta-analysis of 984 endometriosis-related genes identified 39 key endometriosis-related genes, which might be involved with tumour formation [48]. If confirmed, the shared genetic mechanism of cancer and endometriosis might open a new window for diagnosis, risk evaluation, and even treatment. 


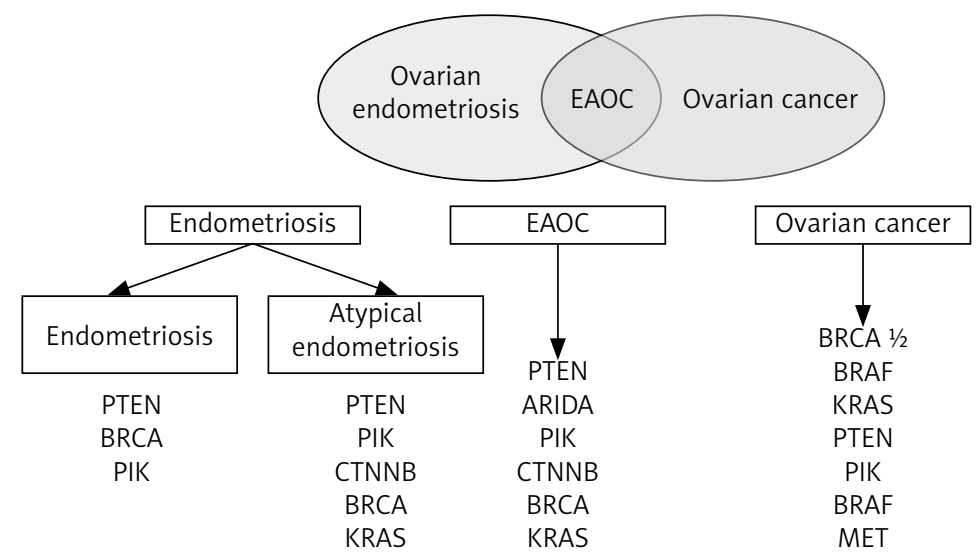

Fig. 4. Genes involved in development of endometriosis, endometriosis-associated ovarian cancer, and ovarian cancer

One of the newest suggestions is focused on Notch signalling, but again, the few studies on this subject yielded controversial results [49]. These differences might be caused by different experimental approaches.

Some interesting hypotheses about the endometriosis-related ovarian cancer have been proposed [50]. One involves extracellular haemoglobin, iron, and heme causing cellular oxidative damage via increased reactive oxygen species with subsequent DNA damage and subsequent mutations. This hypothesis is based on the incessant menstruation theory. The second one suggested the opposite and involves increased production of antioxidants, which might favour a tumour-potentiating environment. Both options are based on the idea of the double-edged sword of redox imbalance [51]. Additional risk factors might involve age over 42 years and post-menopause status [52]. The authors underline the need for better evaluation of these risks. Age is clearly the main risk factor.

Another possibility involves the immune system. Endometriosis-related cancers often have distinct immune profiles that significantly differ from de novo tumours. At the same time, many aspects of the defence system are changed in endometriosis, including macrophages, lymphocytes, and natural killer cell functions [53], leading to the hypothesis of the involvement of a changed immune system in ovarian cancer progression. However, it is still not fully established whether immunological changes found in patients with endometriosis are the cause or a secondary result.

\section{Endometrial cancer}

Endometrial cancer is common but has better prognosis, with the overall 5 -year survival rate reaching almost $85 \%$ [54]. Some studies, similarly to ovarian cancer, suggest an association between endometriosis and endometrial cancer with a possible significantly higher rate [34].
In endometrial cancer, apoptosis is often considered to be responsible for the development of this type of cancer. With a rate 3 times above control values, KARAS LC56 polymorphism might be one of the factors involved in the development and progression of this disease [55], but the low numbers of patients evaluated in this study make any conclusion questionable. A recent study showed that apoptosis inducer apoptosis-stimulating p53 protein 2 (ASPP2) might be strongly involved because its suppression promoted malignancy. The exact mechanism of action showed that ASPP2 acts via Yes-associated protein and lipolysis-stimulated lipoprotein receptor [56]. If these in vitro experiments are further confirmed, ASPP2 might be developed into a therapeutic target. However, new diagnostic or therapeutic processes are lacking [6].

\section{Conclusions}

Both endometriosis and ovarian cancer are multifactorial diseases, so it is not surprising that even after decades of intensive research, more questions than answers remain. The evidence suggesting that patients with endometriosis have a higher risk of developing ovarian cancer is interesting but unclear because the data are often contradictory. One of the reasons for such a discrepancy might be the fact that most of the results are from retrospective cohort studies of hospitalized patients. The vast amount of these studies offers little actual information because the results differ widely based on exclusion criteria.

The mechanisms resulting in malignant transformation are unclear, and the fact that benign or at least benign-appearing ovarian masses are often detected several years before cancer diagnosis [57] makes the situation more problematic. Even more inconclusive is the question of the extent to which endometriosis-ovarian cancer is causative. Somatic mutations of PIK3CA, PTEN, and ARID1A might play a role in the disease progression and malignant transformation. 
The list of potential molecules that might be involved is rather long but is almost always based on a simple comparison of the levels of expression in patients with endometriosis and ovarian (or other) cancer. To some extent, it is almost certain that a detailed study will find differences in the levels of some molecules, making these results interesting but clinically questionable. The possible mechanisms of action remain, however, at the theoretical level only. At present, not a single marker can be used for diagnosis, let alone treatment. Even though endometriosis is a serious health problem, the incidence of ovarian cancer is 3 times higher after in vitro fertilization [58]. In addition, the question of how to identify patients diagnosed with endometriosis who might be at high risk of developing ovarian cancer remains critical but unanswered.

Clearly more research and fundamentally new experimental approaches are necessary for better understanding and treatment of endometriosis and endometriosis-related morbidity.

\section{Disclosure}

The authors report no conflict of interest.

\section{References}

1. Kralickova M, Vetvicka V, Fiala L, et al. The search for biomarkers in endometriosis: a long and windy road. Reprod Sci 2021.

2. Kajihira H, Yamada Y, Kanayama S, et al. New insight into the pathology of endometriosis: from chronic inflammation to danger signal. Gynecol Endocrinol 2011; 2: 73-79.

3. Szamatowicz M, Hermanowicz-Szamatowicz K. Endometriosis - a decade later - still an enigmatic disease. What is the new in the diagnosis and treatment? Oncol Endocrinol 2020; 36: 104-108.

4. Lagana AS, Garzon S, Gotte M, et al. The pathogenesis of endometriosis: molecular and cell biology insights. Int J Mol Sci 2019; 20: 5615.

5. Sampson JA. Endometrial carcinoma of the ovary arising in endometrial tissue in that organ. Arch Surg 1925; 10: 1-72.

6. Scott RB. Malignant change in endometriosis. Obstet Gynecol 1953; 2: 283-289.

7. Kralickova M, Vetvicka V, Lagana AS. Endometrial cancer - is our knowledge changing? Transl Cancer Res 2020; 9: 7734-7745.

8. Pearce CL, Templeman C, Rossing MA, et al. Association between endometriosis and risk of histological subtypes of ovarian cancer: a pooled analysis of case-control studies. Lancet Oncol 2012; 13: 385-394.

9. Levin G, Cheng C, Healey M, et al. Endometriosis - it is not just beginning. J Gynecol Obste Human Reprod 2020; 49: 101744.

10. Olovsson M. Immunological aspects of endometriosis: An update. Am J Reprod Immunol 2011; 66 (Suppl.1): 101-114.

11. Vlahos NF, Kalampokas T, Fotiou S. Endometriosis and ovarian cancer. A review. Gynecol Endocrin 2010: 26: 213-219.

12. Hunn, J, Rodriquez GC. Ovarian cancer: etiology, risk factors, and epidemiology. Clin Obstet Gynecol 2012; 1: 3-23.

13. Ralaitzopoulos DR, Mitsopoulou A, Illiopoulou SM, et al. Association between endometriosis and gynecological cancers: a critical review of the literature. Arch Gynecol Obstetr 2020; 301: 355-367.

14. Ota Y, Ota K, Takahashi T, et al. Primary endometrioid carcinoma of the uterosacral ligament arising from deep infiltrating endometriosis 6 years after bilateral salphingo-oophorectomy due to atypical proliferative endometrioid tumor of the ovary: a rare case report. World J Surg Oncol 2020; 18: 329.
15. Pan Q, Luo X, Toloubeydokhti T, et al. The expression profile of microRNA in endometrium and endometriosis and the influence of ovarian steroids on their expression. Mol Hum Reprod 2007; 13: 797-806.

16. Nakamura N, Terai $\mathrm{Y}$, Nunode $\mathrm{M}$, et al. The differential expression of miRNAs between ovarian endometrioma and endometriosis-associated ovarian cancer. J Ovar Canc 2020; 13: 51.

17. Suryawanshi S, Vlad AM, Lin HM, et al. Plasma microRNAs as novel biomarkers for endometriosis and endometriosis-associated ovarian cancer. Clin Cancer Res 2013; 19: 1213-1224.

18. Wu RL, Ali S, Bandyopadhyay S, et al. Comparative analysis of differentially expressed miRNAs and their downstream mRNAs in ovarian cancer and its associated endometriosis. J Cancer Sci Ther 2015; 7: 258-265.

19. Kralickova M, Vetvicka V. Endometriosis and ovarian cancer. World J Clin Oncol 2014; 5: 800-805.

20. Gadducci A, Zannoni GF. Endometriosis-associated extraovarian malignancies: a challenging question for the clinician and the pathologist. Anticancer Res 2020; 40: 2429-2438.

21. Hermens $M$, van Altena AM, van der Aa M, et al. Ovarian cancer prognosis in women with endometriosis: a retrospective nationwide cohort study of 32,419 women. Am J Obst Gynecol 2021; 224: 284.e1-284.e10.

22. Hermens $M$, van Altena AM, Niebort TE, et al. Incidence of endometroid and clear-cell ovarian cancer in histological proven endometriosis: the ENOCA population-based cohort study. Am J Obstet Gynecol 2020; 223: 107.e1-11.

23. Buis CC, van Leeuwen FE, Mooiji TM, et al. OMEGA Project Group. Increased risk for ovarian cancer and borderline ovarian tumours in subfertile women with endometriosis. Hum Reprod 2013; 28: 3358-3369.

24. Pearce CL, Templeman C, Rossing MA, et al. Association between endometriosis and risk of histological subtypes of ovarian cancer: a pooled analysis of case-control studies. Lancet Oncol 2012; 13: 385-394.

25. Dzatic-Smiljkovic O, Vasiljevic M, Djukic M, et al. Frequency of ovarian endometriosis in epithelial ovarian cancer patients. Clin Exp Obstet Gynecol 2011; 38: 394-398.

26. La Grenade A, Silverberg SG. Ovarian tumors associated with atypical endometriosis. Hum Pathol 1988; 19: 1080-1084.

27. Ness RB, Cottreau C. Possible role of ovarian epithelial inflammation in ovarian cancer. J Natl Cancer Inst 1999; 91: 1459-1467.

28. Wang C, Liang Z, Liu X, et al. The association between endometriosis, tubal ligation, hysterectomy and epithelial ovarian cancer: meta-analyses. Int J Environ Res Public Health 2016; 13: 1138.

29. Kobayashi H. Potential scenarios leading to ovarian cancer arising from endometriosis. Redox Rep 2016; 21: 119-126.

30. Murakami K, Kotani Y, Nakai H, et al. Endometriosis-associated ovarian cancer: the origin and targeted therapy. Cancers 2020; 12: 1676.

31. Brinton LA, Sakoda LC, Sherman ME, et al. Relatioship of benign gynecologic diseases to subsequent risk of ovarian and uterine tumors. Cancer Epidemiol Biomarker Prev 2005; 14: 2929-2935.

32. Sayasneh A, Tsivos D, Crawford R. Endometriosis and ovarian cancer: a systematic review. ISRN Obstet Gyn 2011; 2011: 140310.

33. Kondi-Pafiti A, Papakonstantinou E, lavazzo C, et al. Clinicopathological characteristics of ovarian carcinomas associated with endometriosis. Arch Gynecol Obstet 2012; 285: 479-483.

34. Pejovic T, Thisted $S$, White $M$, et al. Endometriosis and endometriosisassociated ovarian cancer (EAOC). Adv Exp Med Biol 2020; 1242: 73-87.

35. Wei J, William J, Bulun S. Endometriosis and ovarian cancer: a review of clinical, pathologi, and molecular aspects. Int J Gynecol Pathol 2011; 20: 553-568.

36. Kumar S, Munkarah A, Arabi H, et al. Prognostic analysis of ovarian cancer associated with endometriosis. Am J Obstet Gynecol 2011; 204: 63.e1.

37. Kok VC, Tsai HJ, Su CF, et al. The risks for ovarian, endometrial, breast, colorectal, and other cancers in women with newly diagnosed endometriosis or adenomyosis: a population-based study. Int I Gynecol Cancer 2015; 25: 968-976.

38. Chiang AJ, Chang C, Huang CH, et al. Risk factors in progression from endometriosis to ovarian cancer: a cohort study based on medical insurance data. J Gynecol Oncol 2018; 29: e28.

39. Eoh KJ, Han M, Kim EH, et al. Markedly increased risk of malignancies in women with endometriosis. Gynecol Oncol 2021; 161: 291-296. 
40. Bertelsen L, Mellemkjaer L, Frederiksen K, et al. Risk for breast cancer among women with endometriosis. Int J Cancer 2007; 120: 1372-1375.

41. Lu J, Tao X, Zhou J, et al. Improved clinical outcomes of patients with ovarian carcinoma arising in endometriosis. Oncotarget 2017; 8: 5843-5852.

42. Koiyama S, Aoki D, Tominagha E, et al. Prognosis of Japanese patients with ovarian clear cell carcinoma associated with pelvic endometriosis. Clinicopathologic evaluation. Gynecol Oncol 1999; 72: 342-346.

43. Noli S, Cipriani S, Scarfone G, Villa A, et al. Long term survival of ovarian endometriosis associated clear cell and endometriod ovarian cancers. Int J Gynecol Canc 2013; 23: 244-248.

44. Bounous VE, Ferrero A, Fuso L, et al. Endometriosis-associated ovarian cancer: a distinct clinical entity? Anticancer Res 2016; 36: 3445-3449.

45. Shin HY, Yang W, Chay DB, et al. Tetraspanin 1 promotes endometriosis leading to ovarian clear cell carcinoma. Mol Oncol 2021; 15: 987.

46. Wilbur MA, Shih IM, Segars JH, et al. Cancer implications for patients with endometriosis. Semin Reprod Med 2017; 35: 110-116.

47. Wiegand KC, Shah SP, Al-Agha OM, et al. ARID1A mutations in endometriosis-associated ovarian carcinomas. N Eng J Med 2010; 363: 15321543.

48. Bhyan SB, Zhao L, Wee YK, et al. Genetic links between endometriosis and cancer in women. Peer J 2019; 7: e8135.

49. Jonusiene V, Sasnauskiene A. Notch and endometrial cancer. Exp Med Biol 2021; 1287: 47-57.

50. Kobayashi H. Potential scenarios leading to ovarian cancer arising from endometriosis. Redox Rep 2016; 21: 119-126.

51. Fontana J, Zima M, Vetvicka V. Biological markers of oxidative stress in cardiovascular diseases: after so many studies, what do we know? Immunol Invest 2018; 47: 823-843.

52. Huang CY, Cheng NM, Wang, PH. Risk factors associated with epithelial ovarian cancer in women with endometriosis. Taiwanese J Obstet Gynecol 2020; 59: 353-355.

53. Kralickova M, Vetvicka V. Immunological aspects of endometriosis: a review. Ann Transl Med 2015; 3: 153.

54. Vogel TJ, Knickerbocker A, Shah CA, et al. An analysis of current treatment practice in uterine papillary serous and clear cell carcinoma at two high volume cancer centers. J Gynecol Oncol 2015; 26: 25-31.

55. Cintesun FNI, Kerumoglu OS, Cintesun E, et al. The relation between KRAS LCS6 polymorphism and endometrium cancer. J Obstet Gynecol 2020; 40: 988-993.

56. Konno T, Kohno T, Okada T, et al. ASPP2 suppression promotes malignancy via LSR and YAP in human endometrial cancer. Histochem Cell Biol 2020; 154: 197-213.

57. Kobayashi H. Ovarian cancer in endometriosis: epidemiology, natural history, and clinical diagnosis. Int J Clin Oncol 2009; 14: 378-382.

58. Van Leeuwen FE, Klip H, Mooij TM, et al. Risk of borderline and invasive ovarian tumours after ovarian stimulation for in vitro fertilization in a large Dutch cohort. Human Reprod 2011; 16: 3456-3465. 